\title{
Variação temporal e espacial na composição de guildas alimentares da ictiofauna em lagoas marginais do Rio Cuiabá, Pantanal Norte
}

\author{
Lidiani Queli Lubas Ximenes ${ }^{1,4}$, Lúcia Aparecida de Fatima Mateus ${ }^{2}$ \& Jerry Magno Ferreira Penha \\ ${ }^{\text {I} P r o g r a m a ~ d e ~ P o ́ s-G r a d u a c ̧ a ̃ o ~ e m ~ E c o l o g i a ~ e ~ C o n s e r v a c ̧ a ̃ o, ~ U n i v e r s i d a d e ~ F e d e r a l ~ d e ~ M a t o ~ G r o s s o ~ d o ~ S u l-~}$ \\ UFMS, CEP 79070- 900, Campo Grande, MS, Brasil,e-mail: lqueli@hotmail.com \\ ${ }^{2}$ Laboratório de Ecologia e Manejo de Recursos Pesqueiros, Instituto de Biociências, Universidade Federal \\ de Mato Grosso-UFMT, CEP 78060-900,Cuiabá,MT,Brasil,e-mail:lmateus@ufmt.br \\ ${ }^{3}$ Laboratório de Ecologia de Populações, Instituto de Biociências, Universidade Federal de Mato Grosso - \\ UFMT, CEP 78060-900, Cuiabá, MT, Brasil,e-mail: jpenha@ufmt.br \\ ${ }^{4}$ Autor para correspondência: Lidiani Queli Lubas Ximenes, e-mail: lqueli@hotmail.com
}

XIMENES, L.Q.L., MATEUS, L.A.F. \& PENHA, J.M.F. Temporal and Spatial variation in composition of feeding guilds of the fish fauna of oxbow lakes of the Cuiabá River, Northern Pantanal. Biota Neotrop. 11(1): http://www.biotaneotropica.org.br/v11n1/en/abstract?article+bn03211012011.

\begin{abstract}
This study aimed to evaluate the spatial and temporal variation of the feeding guilds of fish communities of 10 oxbow lakes of the Cuiabá River in the Pantanal. The lagoons were sampled in three periods of the hydrological cycle (beginning and ending of the dry and flooding season of 2005). The feeding guilds were determined through analysis of stomach contents of the most abundant species in the community. Were analyzed 37 species belonging to eight trophic guilds (insectivore, herbivore, omnivore, zooplanktivore, planktivore, detritivore, benthivore and iliophage), whitch varied spatially and temporally. A change in the composition of trophic guilds between the periods and locations was observed, but the number of guilds did not vary for either of them. Our results suggest that spatial and temporal variation in the composition of the guilds can be related to many factors, such as alterations in the composition of the community where new species can contribute to the different trophic guilds; and the exploration of more abundant resources in certain periods of the year favoring the presence of some guilds only in some periods.
\end{abstract}

Keywords: trophic groups, fishes, hydrological cycle, feeding ecology, community structure.

XIMENES, L.Q.L., MATEUS, L.A.F. \& PENHA, J.M.F. Variação temporal e espacial na composição de guildas alimentares da ictiofauna em lagoas marginais do Rio Cuiabá, Pantanal Norte. Biota Neotrop. 11(1): http://www.biotaneotropica.org.br/v11n1/pt/abstract?article+bn03211012011.

Resumo: O presente trabalho teve como objetivo avaliar a variação espacial e temporal das guildas alimentares das comunidades de peixes em 10 lagoas marginais do Rio Cuiabá no Pantanal. As lagoas foram amostradas em três períodos do ciclo hidrológico (início e final da seca e início da enchente de 2005). As guildas alimentares foram determinadas através da análise do conteúdo estomacal das espécies mais abundantes da comunidade. Foram analisadas 37 espécies pertencentes a oito guildas alimentares (insetívora, herbívora, onívora, zooplanctívora, planctívora, detritívora, bentívora e iliófaga), as quais variaram espacial e temporalmente. Observamos uma mudança na composição das guildas tróficas entre os períodos e locais analisados, porém o número de guildas não variou espaço-temporalmente. Nossos resultados sugerem que as mudanças espaciais e temporais na composição das guildas podem estar relacionadas com diversos fatores, como as alterações na composição da comunidade onde novas espécies podem compor as diferentes guildas; a exploração de recursos mais abundantes em determinadas épocas do ano favorecendo a presença de algumas guildas somente em alguns períodos.

Palavras-chave: grupos tróficos, peixes, ciclo hidrológico, ecologia alimentar, estrutura de comunidade. 


\section{Introdução}

Registros sobre a alimentação de peixes são comuns em estudos de ecologia de comunidades. Entretanto, determinar os padrões de estrutura alimentar de uma comunidade através das guildas existentes não é uma tarefa fácil já que as espécies apresentam alterações na dieta em função do espaço e do tempo e com o desenvolvimento dos indivíduos. As guildas alimentares presentes no habitat influenciam diretamente o tamanho das cadeias alimentares (Hoeinghaus et al. 2008) afetando a estrutura da comunidade (Pace et al. 2004; Paine 1980) e o funcionamento do ecossistema (Duffy et al. 2005).

O termo "guilda" tem sido bastante utilizado em trabalhos de ecologia de comunidade sendo definido por Simberloff \& Dayan (1991) como um grupo de espécies que exploram os mesmos recursos de forma similar. O uso deste termo em estudos ecológicos deve ser analisado criteriosamente já que há distinção entre o termo guilda e demais termos utilizados como grupos funcionais, guildas tróficas, nichos tróficos. Menge et al. (1986) distinguiram o termo guilda de grupos funcionais definindo a guilda como um grupo de espécies que utilizam o mesmo recurso e grupos funcionais como método de forrageamento das espécies. Neste trabalhou adotou-se a terminologia "guilda trófica" para definir o grupo de espécies que exploram o mesmo recurso alimentar (Yodzis 1982, Burns 1989).

Através da análise das guildas tróficas pode-se descrever a estrutura trófica e as interações alimentares dentro de comunidades biológicas (Specziár \& Rezsu 2009). Além disso, o conhecimento da ecologia trófica de um determinado sistema é importante não só para se determinar os hábitos alimentares das espécies, mas para compreender as relações inter-específicas e inter-guildas (Elliott et al. 2007). De acordo com Winemiller \& Jepsen (1998) esses dados são a base para construção de teias alimentares e fornecem embasamento para trabalhos de manejo e conservação de ecossistemas.

A composição e a distribuição das espécies que compõe as guildas são dependentes de vários fatores como a estrutura do habitat, disponibilidade de alimento, riqueza e composição de espécies da comunidade, a relação inter e intra-específicas e os fatores ambientais. O alimento é o recurso mais comum na segregação entre espécies, sendo que este pode variar no segmento espaço-temporal e afetar diretamente a composição das guildas existentes na comunidade (Ross 1986).

Em áreas alagáveis há períodos de inundação e de seca que influenciam fortemente a estrutura e o funcionamento das comunidades aquáticas devido às condições hidrológicas (Lake 2003). Mudanças temporais na disponibilidade de alimento afetam as relações tróficas da comunidade (Winemiller \& Jepsen 1998), pois alteram a composição qualitativa e quantitativa da oferta de recursos ao longo do ano.

Além disso, a variação espacial no tipo de habitat é outro fator importante que influencia a estratégia alimentar, determinando as oportunidades de forrageamento das espécies (Hajisamae et al. 2003) sendo que a disponibilidade de recursos para uma espécie pode ser diferente conforme o habitat que ela esta inserida (Hajisamae 2009).

Apesar de vários estudos sobre alimentação das espécies de peixes do Pantanal terem sido realizados (Sazima \& Machado 1990, Catella 1992, Resende 1996), a maioria deles procurou apenas identificar os hábitos alimentares das espécies não verificando a influência que as mudanças espaciais e temporais no habitat e nas características dos ambientes estudados têm na composição das guildas alimentares. Desta forma, neste trabalho tivemos como objetivo determinar os padrões de variação das guildas alimentares em lagoas marginais do Rio Cuiabá, procurando indicar quais guildas alimentares compõe a comunidade e avaliando se o número e a composição das guildas alimentares se alteram espaço-temporalmente.

\section{Material e Métodos}

\section{1. Área de estudo}

As coletas foram realizadas em lagoas marginais da planície de inundação do Rio Cuiabá, situadas na Reserva Particular de Patrimônio Natural (RPPN) SESC-Pantanal e seu entorno. A região é situada na porção norte do Pantanal Matogrossense e está posicionada entre os paralelos $16^{\circ}$ e $17^{\circ} \mathrm{S}$ e os meridianos $56^{\circ}$ e $57^{\circ} \mathrm{W}$ no Estado de Mato Grosso (Figura 1).

O ciclo hidrológico da região pode ser dividido em quatro períodos (enchente, cheia, vazante e seca) de acordo com o nível da água do rio. Dados históricos (1993-2004) indicam que no Rio Cuiabá o inicio da inundação na planície ocorre quando o nível do rio atinge $240 \mathrm{~cm}$, sendo que isso normalmente ocorre entre os meses de dezembro e abril, podendo este período variar entre os anos (Bailly et al. 2008).

As amostragens dos peixes foram realizadas na zona litoral vegetada de 10 lagoas permanentes da planície de inundação do Rio Cuiabá durante o ano de 2005, em três períodos do ciclo hidrológico da região: junho (início da seca), setembro (final da seca) e dezembro (início da enchente) (Figura 2).

Para a coleta dos exemplares foram realizados nove lances de peneira na zona litoral vegetada das lagoas. Esse apetrecho é composto de um saco em forma de funil com $1 \mathrm{~m}^{2}$ de boca e profundidade de $1 \mathrm{~m}$, confeccionado com tela de naylon de $1,5 \mathrm{~mm}$ de abertura de malha. Apesar de ineficiente para captura de espécies de grande porte, é eficiente para a coleta de espécies de pequeno porte, que são predominantes nas zonas litorais vegetadas das lagoas da região (Baginski et al. 2007).

As seguintes características físicas das lagoas foram tomadas: i) proporção de macrófitas aquáticas (obtida visualmente através de um ranking com quatro posições variando de 0 a $100 \%$ de cobertura); ii) a área de superfície das lagoas (hectares) e a distância mínima do rio (metros) obtidas através de imagens do Satélite Landsat7 (Padovani et al. 2003) (Tabela 1).

\section{Análise de dados}

Para determinação da dieta foram analisados 10 conteúdos estomacais das espécies mais abundantes, consideradas aqui como aquelas que apresentaram abundância superior a 10 indivíduos $/ \mathrm{m}^{2} \mathrm{em}$ pelo menos uma lagoa analisada. Os exemplares foram eviscerados e seus conteúdos estomacais analisados sob microscópio estereoscópico. Os recursos alimentares foram identificados com auxilio de bibliografias apropriadas a cada grupo taxonômico: Borror \& Delong (1988), Perez (1988), Stehr (1987) para invertebrados; Needham \& Needham 1982 para organismos aquáticos em geral; Elmoor-Loureiro 1997 para zooplâncton e Bicudo \& Menezes 2006 para o fitoplâncton.

Os recursos alimentares foram quantificados pelo método de pontos (Hynes 1950), a conversão dos itens ingeridos pelas espécies em proporções relativas foi calculada através da relação 
Variação temporal e espacial na composição de guildas tróficas de peixes

Tabela 1. Características principais das lagoas marginais amostradas no Rio Cuiabá, Pantanal Norte.

Table 1. Main characteristics of the sampled oxbow lakes of the Cuiabá River, Northern Pantanal.

\begin{tabular}{|c|c|c|c|c|c|}
\hline Lagoas amostradas & $\begin{array}{c}\text { Distância do rio } \\
(\mathrm{m})\end{array}$ & $\begin{array}{c}\text { Área da lagoa } \\
\text { (ha) }\end{array}$ & Conexão com o rio & $\begin{array}{c}\text { (\%) de macrófitas } \\
\text { aquáticas }\end{array}$ & $\begin{array}{c}\text { Coordenadas } \\
\text { geográficas }\end{array}$ \\
\hline Antônio Alves & 0 & 17,59 & Conectada & 25 & $\begin{array}{l}16^{\circ} 31^{\prime} 32.1^{\prime \prime} \mathrm{S} \\
56^{\circ} 23,24.5 " \mathrm{~W}\end{array}$ \\
\hline Biguás & 589 & 16,9 & Isolada & 25 & $\begin{array}{l}16^{\circ} 31^{\prime} 04.2 ” \mathrm{~S} \\
56^{\circ} 23,22.7^{\prime \prime} \mathrm{W}\end{array}$ \\
\hline Corixão & 0 & 9,28 & Conectada & 75 & $\begin{array}{l}16^{\circ} 33^{\prime} 36.6^{\prime \prime} \mathrm{S} \\
56^{\circ} 24^{\prime} 54.8^{\prime \prime} \mathrm{W}\end{array}$ \\
\hline Estiva & 240,7 & 5,7 & Isolada & 50 & $\begin{array}{l}16^{\circ} 39^{\prime} 05.9^{\prime \prime} \mathrm{S} \\
56^{\circ} 28^{\prime} 46.0^{\prime \prime} \mathrm{W}\end{array}$ \\
\hline Macário & 30 & 14,65 & Isolada & 25 & $\begin{array}{l}16^{\circ} 38^{\prime} 09.0^{\prime \prime} \mathrm{S} \\
56^{\circ} 27^{\prime} 27.9^{\prime \prime} \mathrm{W}\end{array}$ \\
\hline Mussum I & 558 & 1,56 & Isolada & 50 & $\begin{array}{l}16^{\circ} 38^{\prime} 41.3 ” \mathrm{~S} \\
56^{\circ} 28^{\prime} 39.1 ” \mathrm{~W}\end{array}$ \\
\hline Mussum II & 108,97 & 0,92 & Isolada & 75 & $\begin{array}{l}16^{\circ} 38^{\prime} 58.5 " \mathrm{~S} \\
56^{\circ} 28^{\prime} \\
17.6^{\prime \prime} \mathrm{W}\end{array}$ \\
\hline Ninhal & 20 & 7,41 & Isolada & 50 & $\begin{array}{l}16^{\circ} 37^{\prime} 51.2^{\prime \prime} \mathrm{S} \\
56^{\circ} 26^{\prime} 58.8^{\prime \prime} \mathrm{W}\end{array}$ \\
\hline PRC & 248 & 0,14 & Isolada & 100 & $\begin{array}{l}16^{\circ} 40^{\prime} 06.3 ” \mathrm{~S} \\
56^{\circ} 28^{\prime} 37.8^{\prime \prime} \mathrm{W}\end{array}$ \\
\hline Santa Rosa & 150 & 20,3 & Isolada & 25 & $\begin{array}{l}16^{\circ} 41^{\prime} 56.2^{\prime \prime} \mathrm{S} \\
56^{\circ} 28^{\prime} 37.3 ” \mathrm{~W}\end{array}$ \\
\hline
\end{tabular}

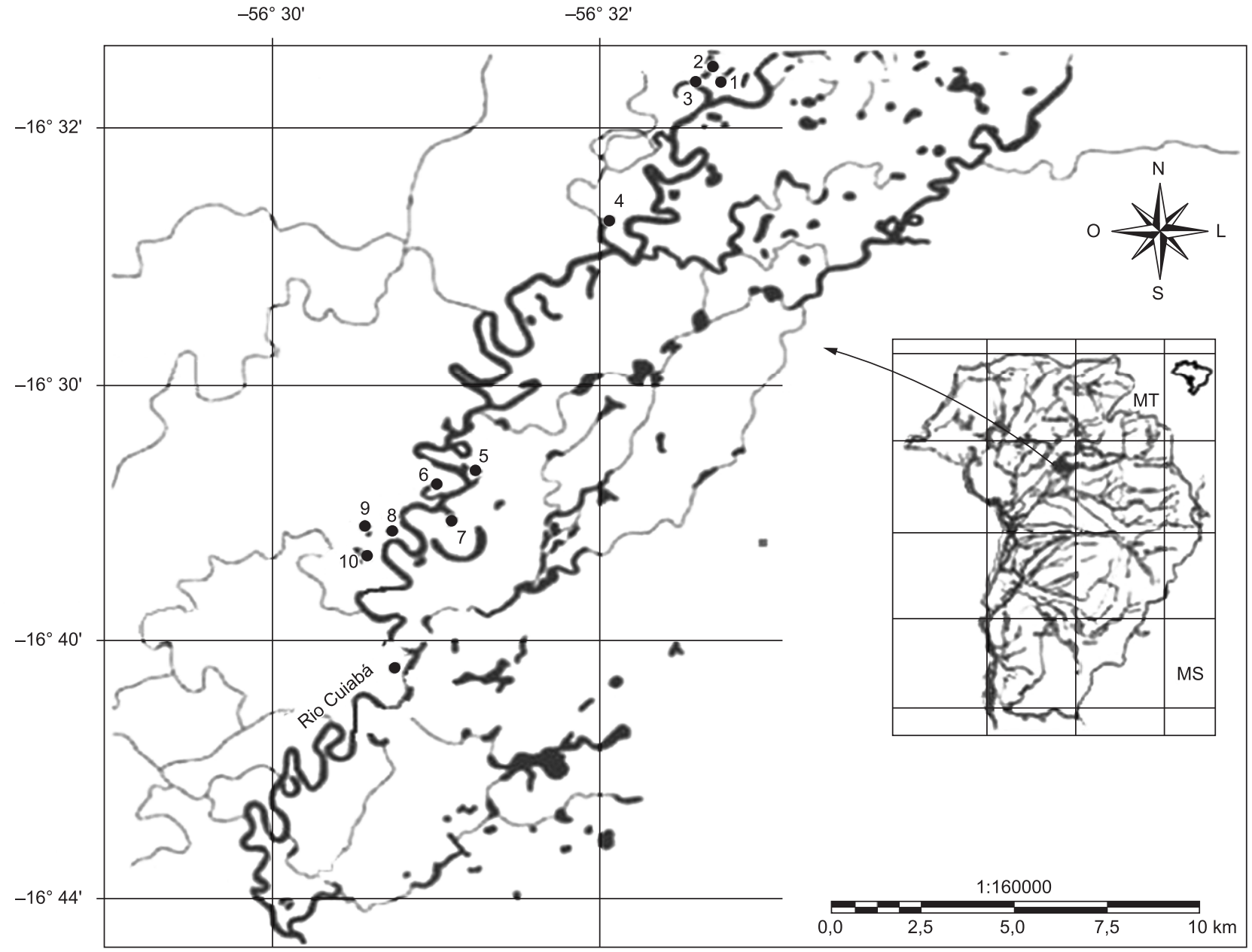

Figura 1. Área de estudo com a localização das lagoas amostradas.

Figure 1. Study area indicating the location of the sampled oxbow lakes. 


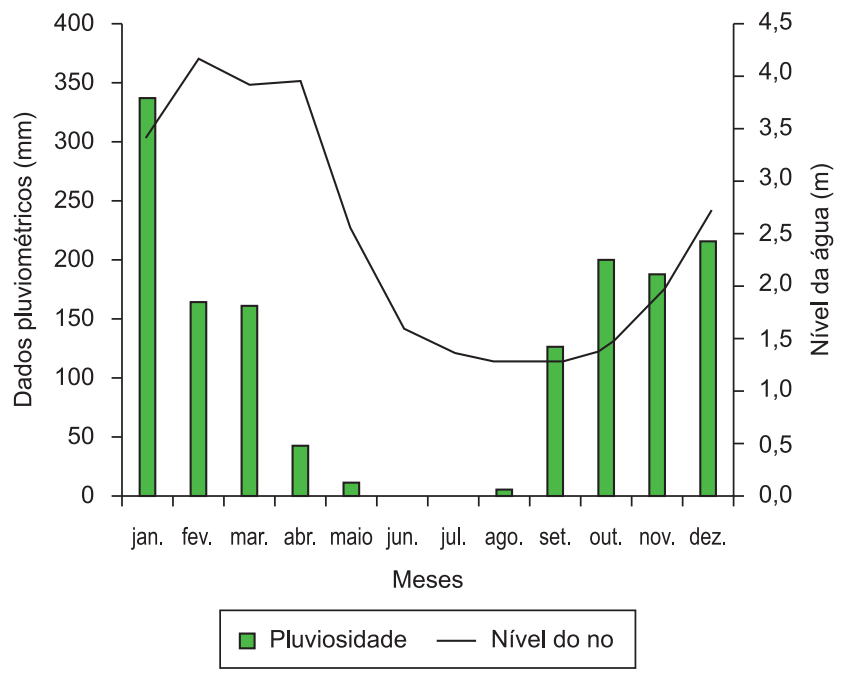

Figura 2. Dados médios de Pluviosidade (mm) e Nível da Água (m) do Rio Cuiabá no ano de 2005. Dados fornecidos pela Agência Nacional de Águas (ANA).

Figure 2. Average precipitation data $(\mathrm{mm})$ and water level $(\mathrm{m})$ of the Cuiabá River in 2005. Data provided by the National Water Agency (ANA).

entre: número de pontos de determinado item ingerido pela espécie i/pontuação total dos recursos ingeridos pela espécie $\mathrm{i}^{*} 100(\mathrm{Pi} / \mathrm{Pt} * 100)$. Os recursos alimentares foram divididos em 14 categorias amplas sendo estas:

- Insetos terrestres: indivíduos inteiros ou restos de insetos terrestres;

- Insetos Aquáticos: estágios imaturos de insetos (larvas, pupas, casulos);

- Detrito: material fino e particulado, constituído de matéria inorgânica, principalmente areia ou silte;

- Sedimento: Material orgânico em diferentes estágios de decomposição;

- Vegetal: Folhas, frutos, sementes e outros vegetais superiores,

- Algas: Algas filamentosas e unicelulares;

- Peixes: Fragmentos ou peixes inteiros, escamas e nadadeiras.

- Protozoários: protozoários e tecamebas;

- Nematódoides: vermes;

- Invertebrados: aranhas e ácaros;

- Fragmentos de insetos: restos de insetos de origem não identificada;

- Moluscos: principalmente bivalves inteiros ou pedaços;

- Zooplâncton: constituído principalmente de cladoceras e copépodas.

- Material não identificado

As espécies foram classificadas em guildas alimentares de acordo com o item predominante na dieta, utilizando-se o total de pontos obtidos por espécie e considerando como critério de inclusão valores $\geq 50 \%$ do espectro alimentar de cada uma delas. A classificação foi realizada conforme Hahn et al. (1998) sendo as espécies distribuídas nas seguintes guildas:

Onívora (O): Peixes que apresentaram um espectro alimentar muito amplo sem predomínio de qualquer recurso em particular, consomem desde algas até vegetais superiores e desde invertebrados a peixes.
- Zooplanctívora $(Z)$ : Espécies que consumiram principalmente cladóceros e copépodos;

- Herbívora $(\mathrm{H})$ : Espécies que ingeriram predominantemente partes vegetais superiores (folhas, sementes e frutos) e algas filamentosas;

- Insetívora(I): Espécies que ingeriram insetos aquáticos e terrestres em diferentes fases de desenvolvimento podendo se alimentar tanto no substrato (principalmente de larvas) como na superfície (insetos terrestres);

- Bentívora (B): Peixes que forragearam principalmente no fundo, ingerindo organismos da fauna bentônica (tendo como recursos predominantes tecamebas, rotíferos, nematóides, microcustáceos, moluscos e pequenas larvas de insetos) e quantidade considerável de sedimento;

- Planctívora (P): Consumiram principalmente fitoplâncton e zooplâncton na dieta;

- Iliófoga (IL): Ingerem grandes quantidades de lodo com matéria orgânica em fases avançadas de dissociação, juntamente com microorganismos e algas a partir de depósitos de fundo ou sobre estratos verticais;

- Detritívora (D): O alimento é também obtido em depósitos de fundo, ingerindo grandes quantidades de matéria orgânica vegetal, porém em fases moderadas de dissociação e acompanhadas de fragmentos de invertebrados. Difere do grupo anterior pelo caráter ausente ou rudimentar do estômago.

A importância dos itens alimentares na dieta de cada espécie foi calculada para todos os períodos e locais analisados, sendo que as espécies foram agrupadas nas guildas alimentares conforme o item predominante na dieta. Esses dados foram alocados em uma matriz de similaridade que foi construída utilizando o índice de Morisita-Horn. Uma Análise de Agrupamento não hierárquica com base no método de ligação UPGMA (Unweighted Pair Group Method with Arithmetic Mean) foi empregada sobre a matriz de similaridade para identificação das guildas. Esse método de ligação é construído com a média aritmética das guildas, dando pesos iguais a todos os objetos. O método de agrupamento descreve o padrão de similaridade entre as espécies, mas pode apresentar um acúmulo de erros a cada ciclo de inclusão de espécies, refletindo no dendograma (Meyer 2002). Assim, a matriz de similaridade foi comparada com uma matriz cofenética para testar possíveis distorções no processo de construção do dendograma. A análise de correlação cofenética é empregada com intuito de aumentar a confiabilidade das conclusões extraídas da interpretação do dendograma (Kopp et al. 2007), como critério de fidelidade nesta análise foi adotado o valor de $\mathrm{r}_{\mathrm{c}}=0,80$ (Romesburg 1985).

Para avaliar se havia diferença significativa entre as guildas identificadas pela análise de agrupamento foi realizada uma Análise de Similaridade (ANOSIM), utilizando o índice de dissimilaridade de Bray-Curtis. Essa análise é uma ferramenta não paramétrica proposta por Clarke (1993), que avalia a existência de diferença significativa na composição das guildas encontradas pelo agrupamento, sendo adotado um nível de significância de 0,05.

Com intuito de avaliar a organização espacial e temporal das guildas alimentares foi calculada a contribuição média de cada recurso na dieta das espécies em cada período do ciclo hidrológico e lagoa. Matrizes resumindo informações sobre os recursos alimentares utilizados pelas espécies nas diferentes lagoas e períodos analisados foram utilizadas em análises de ordenação denominada NMDS (Nonmetric Multidimensional Scaling). A medida de dissimilaridade de Bray - Curtis foi utilizada para a construção da ordenação, uma vez identificados os grupos foi realizada uma Análise de Similaridade 
(ANOSIM) para avaliar a existência de diferenças significativas entre eles $(\mathrm{p}<0,05)$ e as guildas foram identificadas através de uma Análise de Agrupamento (Índice de Morisita - Horn e método de ligação UPGMA).

Com objetivo de testar possíveis diferenças no número de espécies e de guildas alimentares foi realizado o teste não paramétrico de Kruskal - Wallis $(\mathrm{H})$, pois os dados não atenderam à suposição de normalidade dos resíduos exigida pela ANOVA (Sokal \& Rohlf 1995) mesmo depois de transformados. Para análise dos dados foram utilizados os Softwares PAST (versão 1.68) (Hommer et al. 2001) e Systat (versão 10.0) (Wilkinson 2000).

\section{Resultados}

\section{Guildas alimentares}

Foram analisados conteúdos estomacais de 1900 indivíduos pertencentes a cinco ordens, 13 famílias e 37 espécies. As espécies foram classificadas em oito guildas alimentares, que foram estatisticamente diferentes em relação à composição de recursos alimentares consumidos (ANOSIM $\mathrm{R}=0,71 \mathrm{p}=0$, 001). Doze espécies foram caracterizadas como insetívoras $(32,4 \%)$, nove como onívoras $(24,3 \%)$, cinco como herbívoras $(13,5 \%)$, cinco como bentívoras $(13,5 \%)$, duas como detritívoras $(5,4 \%)$, duas como planctívoras $(5,4 \%)$, uma como zooplanctívora $(2,7 \%)$ e uma como Iliófaga (2,7\%) (Tabela 2).

Cada guilda alimentar apresentou a presença de um ou dois recursos que foram dominantes na dieta das espécies componentes. As insetívoras apresentaram predomínio de insetos imaturos, sendo que insetos terrestres foram expressivos somente na dieta de Parauchenipterus galeatus (Linnaeus 1766). Todas as espécies herbívoras analisadas são representantes da Família Characidae (Poptella paraguaensis (Eigenmann 1907), Psellogrammus kennedyii (Eigenmann 1903), Roeboides paranensis Pignalberi 1975, Moenkhausia sanctaefilomenae (Steindachner 1907) e Tetragonopterus argentus Cuvier 1816 e apresentaram dois recursos predominantes na dieta, vegetais e algas.

\section{Variação temporal e espacial nas guildas alimentares}

Encontramos variação espaço-temporal no padrão de uso dos recursos alimentares pela assembléia de peixes e na composição das guildas alimentares. A representação gráfica dos eixos da ordenação evidenciou a separação das guildas nos três períodos considerados (Figura 3) assim como nas diferentes lagoas analisadas (Figura 4) sendo que os eixos do NMDS capturaram grande parte da variação (88,4\%; Stress 0,18 para análise temporal e $81,3 \%$; Stress 0,21 para análise espacial) sendo constatada diferença na composição da dieta das espécies que formaram os grupos (ANOSIM $R=0,29 p=0,001$ para análise temporal e $\mathrm{R}=0,91 \mathrm{p}=0,003$ para análise espacial).

Em relação à variação temporal, observamos uma mudança na composição das espécies que formaram as guildas ao longo do ciclo hidrológico. Das 37 espécies analisadas apenas dez ocorreram em todos os períodos (início e final da seca e início da enchente). No início da seca foram observadas 30 espécies distribuídas em seis guildas alimentares, no final da seca observou-se 22 espécies pertencentes a seis guildas alimentares, já no período de enchente foram encontradas 18 espécies pertencentes a oito guildas alimentares (Figura 5). O número de espécies que compuseram as guildas alimentares variaram entre os períodos analisados (Kruskal - Wallis $\mathrm{H}=11,65 \mathrm{p}=0,003$ ), porém não observou-se mudanças significativas no número de guildas $(\mathrm{H}=5,62 \mathrm{p}=0,06)$.

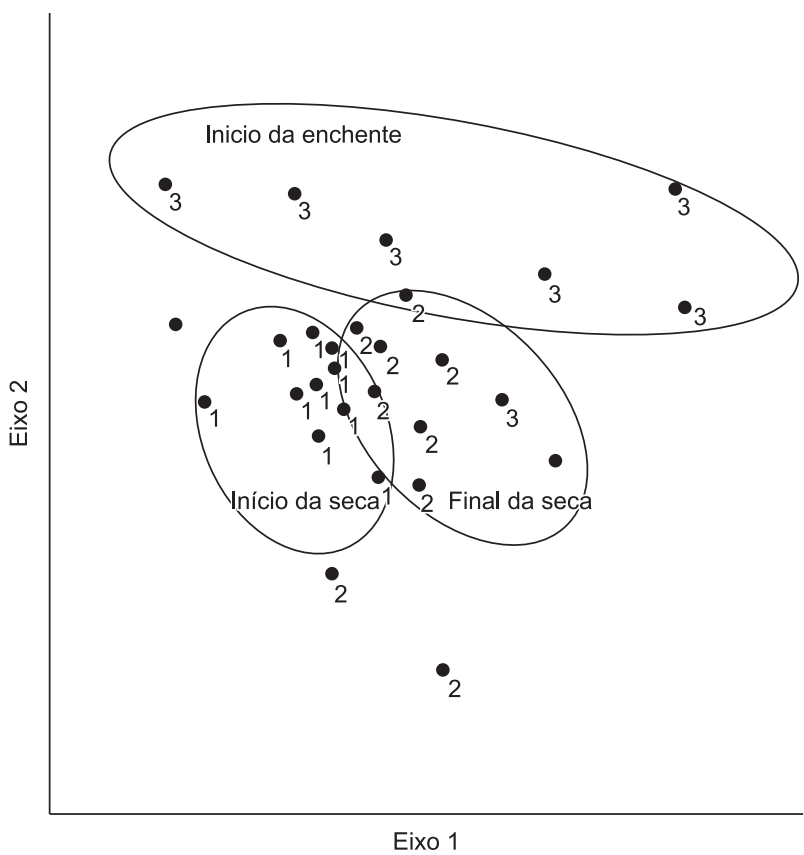

Figura 3. Representação gráfica dos dois primeiros eixos do NMDS, demonstrando a organização trófica temporal dos peixes para as lagoas marginais do Rio Cuiabá (1-Início da Seca, 2- Final da Seca e 3- Início da Enchente).

Figure 3. Graphical representation of the first two axes of the NMDS, demonstrating the temporal trophic organization of fish to the oxbow lakes of the Cuiaba River (1-beginning of the dry, 2- ending of the dry, 3- beginning of the flooding).

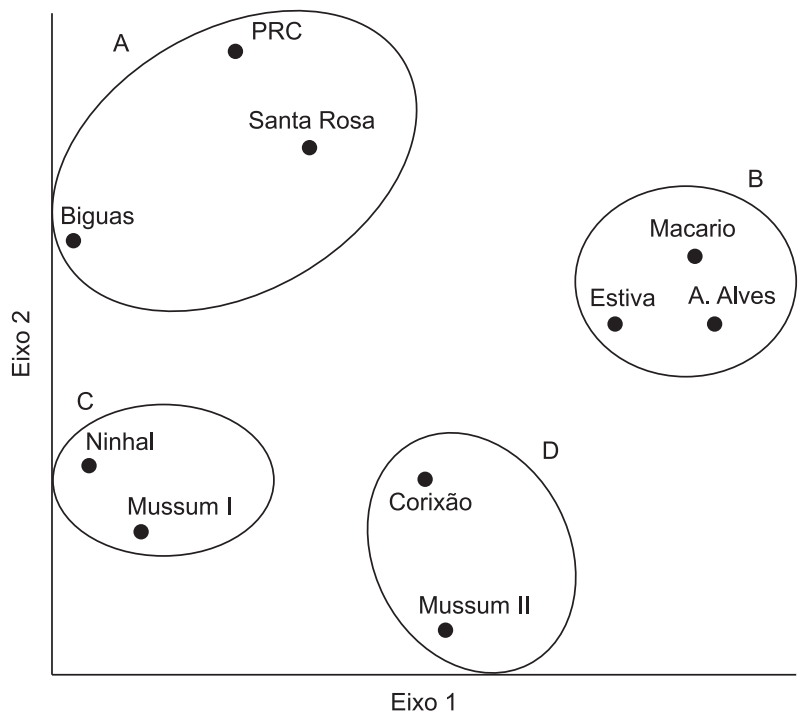

Figura 4. Representação gráfica dos dois primeiros eixos do NMDS, mostrando a organização trófica espacial dos peixes das lagoas marginais do Rio Cuiabá, onde A, B, C e D são os grupos formados pelas lagoas analisadas.

Figure 4. Graphical representation of the first two axes of the NMDS, showing the spatial trophic organization of fish to the oxbow lakes of the Cuiabá River, where A,B, C, D are the groups formed by the studied oxbow lakes. 

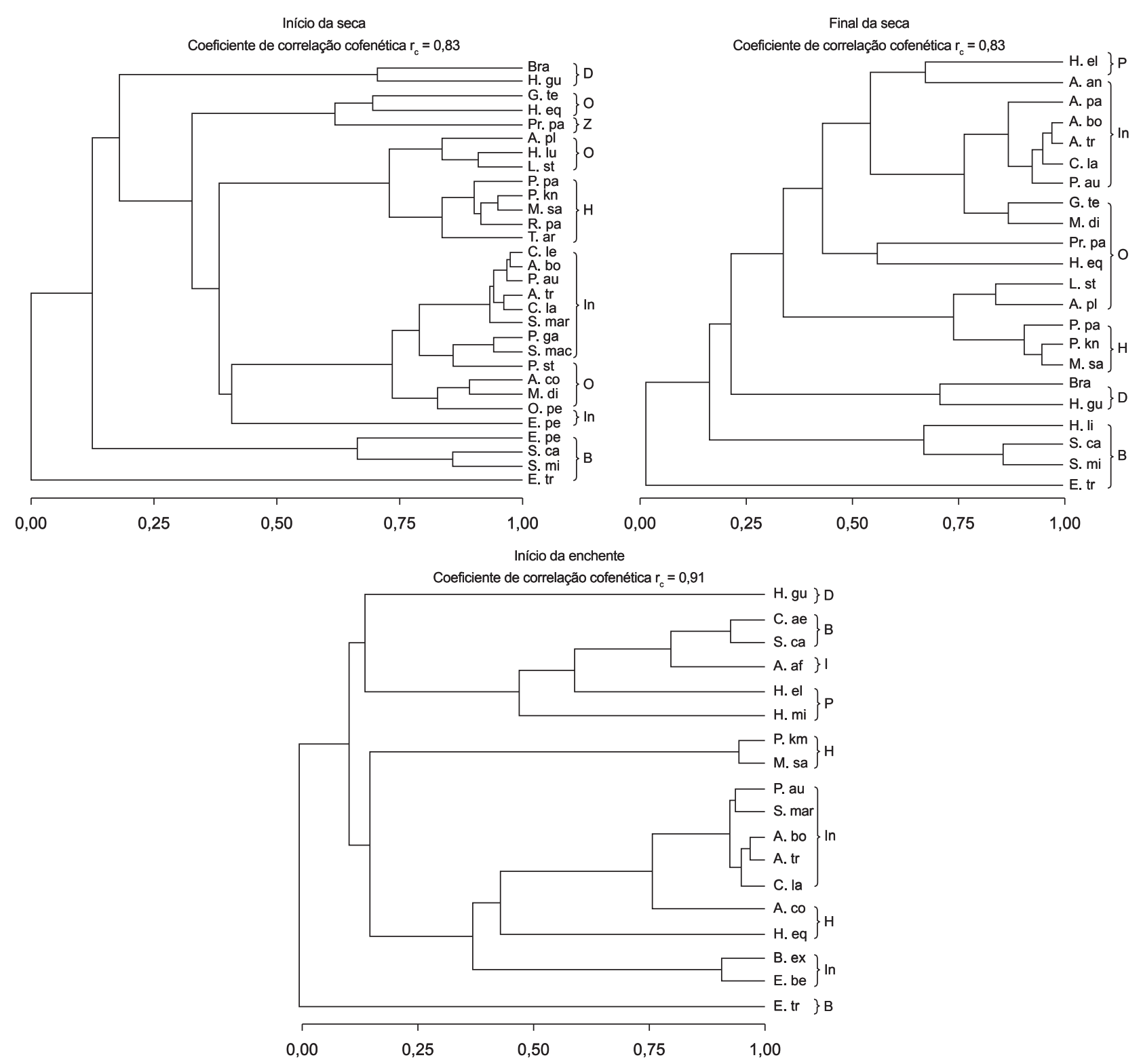

Figura 5. Análise de agrupamento demonstrando as guildas alimentares pertencentes aos grupos encontrados pela representação gráfica do NMDS realizado para os períodos do ciclo hidrológico. (D-Detritívora, O-Onívora, Z-Zooplanctívora, H -Herbívora, In -Insetívora, B-Bentívora, P-Planctívora, I-Iliófaga).

Figure 5. Cluster analysis showing the feeding guilds in the groups found by the graphical representation of NMDS performed for the periods of the hydrological cycle. (D -Detritivore, O -Omnivore, Z-Zooplanktivore, H -Herbivore, In -Insectivore, B -Benthivore, P -Planktivore, I - Illiophage).

Observou-se uma diminuição no número de espécies insetívoras no final da seca e no início da enchente, o mesmo ocorreu com a herbívora. A guilda onívora diminuiu sua riqueza no início da enchente sendo observadas apenas duas espécies pertencentes a essa guilda, já a guilda alimentar iliófoga só ocorreu neste período. Não observamos alterações na composição das guildas bentívora e detritívora entre os períodos analisados. A guilda zooplanctívora ocorreu somente no início da seca e não foram encontradas espécies planctívoras neste período (Figura 5).

Espacialmente observamos que as características das lagoas influenciaram na determinação da composição das guildas. Os grupos foram compostos de diferentes guildas e as que ocorreram em todos os locais variaram em sua composição. O grupo A formado somente por lagoas sem conexão com o corpo d'água, apresentou
24 espécies distribuídas em cinco guildas alimentares, sendo que a maior parte das espécies herbívoras foram observadas neste grupo. No grupo $\mathrm{C}$ formado por lagoas com extensão e cobertura de macrófitas aquáticas mediana e isoladas do corpo d'água observou-se 25 espécies distribuídas em sete guildas alimentares, a guilda zooplanctívora ocorreu somente neste grupo e as guildas insetívora e bentívora tiveram maior abundancia nestes locais. O grupo D foi formado por lagoas com maior porcentagem de macrófitas aquáticas, com 25 espécies distribuídas em sete guildas alimentares foi único grupo com espécie iliófaga (Figura 6). Não houve variação no número de espécies $(H=1,258 \mathrm{p}=0,739)$, de recursos alimentares $(\mathrm{H}=6,42 \mathrm{p}=0.09)$ e de guildas tróficas $(\mathrm{H}=0,225 \mathrm{p}=0,973)$ entre os habitas analisados. 
Variação temporal e espacial na composição de guildas tróficas de peixes

Tabela 2. Recurso principal e guilda alimentar das espécies de peixes. (Veg) Vegetal; (Ins) Insetos; (Sed) Sedimento; (Alg) Algas; (Det) Detrito; (FB) Fauna Bentônica; (Zoo) Zooplâncton; (Fito) Fitoplâncton; (Prot) Protozoários.

Table 2. Principal resource and feeding guild of fish species. (Veg) vegetal; (Ins) Insects; (Sed) Sediment, (Alg) Algae; (Det) Detritus; (FB) Benthic fauna; (Zoo) Zooplankton; (Fito) Phytoplankton; (Prot) Protozoa.

\begin{tabular}{|c|c|c|}
\hline Ordem/família/espécie & Item principal & Guilda trófica \\
\hline \multicolumn{3}{|l|}{ CHARACIFORMES } \\
\hline \multicolumn{3}{|l|}{ Anastomidae } \\
\hline Leporinus striatus Kner, 1858 & Ins/Veg & Onívora \\
\hline \multicolumn{3}{|l|}{ Characidae } \\
\hline Aphyocharax anisitsi Eignenmann \& Kennedy,1903 & Ins & Insetívora \\
\hline Aphyocharax paraguayensis Eignenmann, 1915 & Ins & Insetívora \\
\hline Bryconamericus exodon Eignenmann, 1907 & Ins & Insetívora \\
\hline Gymnocorymbus ternetzi (Boulenger, 1895) & Zoo/Ins/Veg & Onívora \\
\hline Hemigrammus lunatus Durbin, 1918 & Veg/Ins & Onívora \\
\hline Hyphessobrycon elachys (Weitzman, 1984) & Zoo/Fito & Planctívora \\
\hline Hyphessobrycon eques (Steindachner, 1882) & Ins/Veg & Onívora \\
\hline Moenkhausia dichroura (Kner, 1858) & Ins/Alg/Veg & Onívora \\
\hline Moenkhausia sanctaefilomenae (Steindachner,1907) & Veg/Alg & Herbívora \\
\hline Odontostilbe pequira (Steindachner, 1882) & Ins/FB/Veg & Onívora \\
\hline Poptella paraguayensis (Eigenmann, 1907) & Veg/Alg & Herbívora \\
\hline Prionobrama paraguayensis (Eigenmann, 1914) & Zoo & Zooplanctívora \\
\hline Psellogrammus kennedyi (Eigenmann, 1903) & Veg/Alg & Herbívora \\
\hline Roeboides paranensis Pignalberi, 1975 & Veg/Alg & Herbívora \\
\hline Serrapinus calliurus (Boulenger, 1900) & $\mathrm{Sed} / \mathrm{FB}$ & Bentívora \\
\hline Serrapinnus microdon (Eigenmann, 1915) & $\mathrm{Sed} / \mathrm{FB}$ & Bentívora \\
\hline Tetragonopterus argentus Cuvier, 1816 & Veg/Alg & Herbívora \\
\hline \multicolumn{3}{|l|}{ Crenuchidae } \\
\hline Characidium lateralis (Boulenger,1895) & Ins & Insetívora \\
\hline \multicolumn{3}{|l|}{ Hemiodontidae } \\
\hline Hemiodopsis microlepis (Kner, 1858) & Zoo/Sed/Alg & Planctívora \\
\hline Lebiasinidae & & \\
\hline Pyrrhulina australis Eigenmann \& Kennedy, 1903 & Ins & Insetívora \\
\hline \multicolumn{3}{|l|}{ Parodontidae } \\
\hline Apareiodon affinis (Steindachner, 1879) & Sed/Prot & Iliófaga \\
\hline \multicolumn{3}{|l|}{ SILURIFORMES } \\
\hline \multicolumn{3}{|l|}{ Auchenipteridae } \\
\hline Entomocorus benjamini Eignenmann, 1917 & Ins & Insetívora \\
\hline Parauchenipterus galeatus (Linnaeus,1758) & Ins & Insetívora \\
\hline Parauchenipterus striatulus (Steindachner,1876) & Veg/Ins & Onívora \\
\hline \multicolumn{3}{|l|}{ Callichthyidae } \\
\hline Corydoras aeneus (Gill, 1858) & $\mathrm{Sed} / \mathrm{FB}$ & Bentívora \\
\hline Hoplosternum littorale (Hancock, 1828) & Sed/Ins & Bentívora \\
\hline \multicolumn{3}{|l|}{ Loricariidae } \\
\hline Hypoptopoma guentheri Boulenger, 1895 & Det & Detritívora \\
\hline \multicolumn{3}{|l|}{ PERCIFORMES } \\
\hline \multicolumn{3}{|l|}{ Cichlidae } \\
\hline Aequidens plagiozonatus Kullander,1984 & Veg/Ins & Onívora \\
\hline Apistogramma borellii (Regan, 1906) & Ins & Insetívora \\
\hline Apistogramma commbrae (Regan, 1906) & Ins/Alg & Onívora \\
\hline Apistogramma trifasciata (Eignenmann \& Kennedy, 1903) & Ins & Insetívora \\
\hline Crenicichla lepidota Heckel, 1840 & Ins & Insetívora \\
\hline \multicolumn{3}{|l|}{ GYMNOTIFORMES } \\
\hline \multicolumn{3}{|l|}{ Hypopomidae } \\
\hline Brachyhypopomus sp & Det/Ins & Detritívora \\
\hline \multicolumn{3}{|l|}{ Sternopygidae } \\
\hline Eigenmannia trilineata Lopes \& Castello, 1966 & $\mathrm{Sed} / \mathrm{FB}$ & Bentívora \\
\hline Sternopygus macrurus (Boch \&Schneider, 1801) & Ins & Insetívora \\
\hline \multicolumn{3}{|l|}{ SYMBRANCHIFORMES } \\
\hline \multicolumn{3}{|l|}{ Symbranchidae } \\
\hline Synbranchus marmoratus Bloch, 1795 & Ins & Insetívora \\
\hline
\end{tabular}



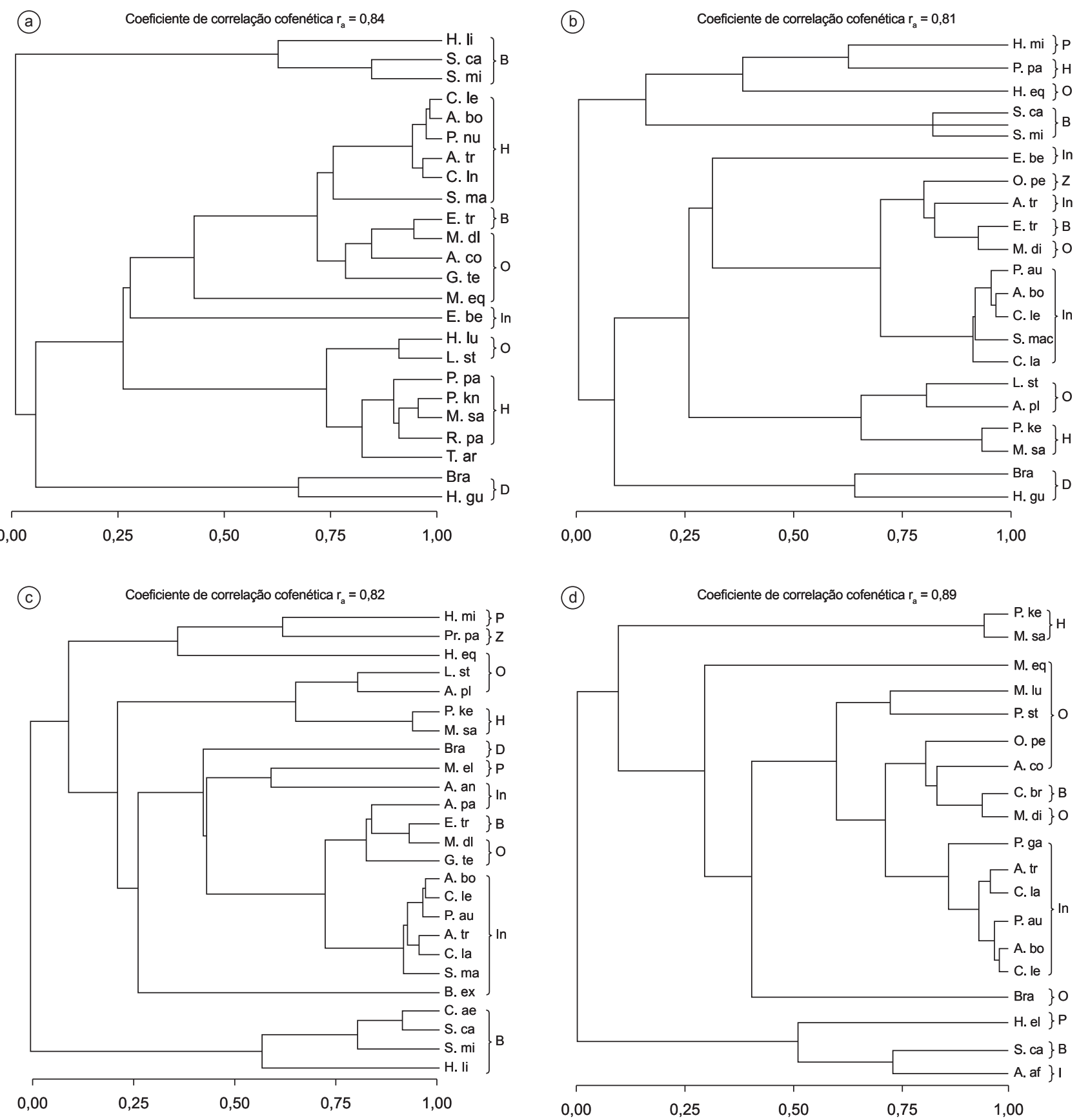

Figura 6. Análise de agrupamento mostrando as diferentes guildas alimentares pertencentes aos grupos encontrados pela representação gráfica do NMDS realizado para os locais analisados. (D-Detritívora, O-Onívora, Z-Zooplanctívora, H -Herbívora, In -Insetívora, B-Bentívora, P-Planctívora, I-Iliófaga).

Figure 6. Cluster analysis showing the feeding guilds in the groups found by the graphical representation of NMDS performed for the locations of the hydrological cycle. (D -Detritivore, O -Omnivore, Z-Zooplanktivore, H -Herbivore, In -Insectivore, B -Benthivore, P -Planktivore, I - Illiophage).

\section{Discussão}

A influência dos componentes espaciais e temporais na disponibilidade dos recursos no ambiente pode levar a uma grande versatilidade na forma de exploração dos mesmos, alterando a ocupação dos nichos ecológicos disponíveis e influenciando diretamente a formação das guildas alimentares. Nossos resultados mostram que algumas guildas são ocupadas por diversas espécies, sendo que essa variação na abundância de espécies entre as guildas analisadas pode ser explicada pelo fato de que alguns recursos são mais explorados pela comunidade, devido: i) à capacidade do recurso ser potencialmente explorado por distintas espécies; ii) à sua maior disponibilidade no ambiente; e iii) à facilidade de sua captura (Abelha et al. 2001, Grenouillet \& Pont 2001, Deus \& Petrere Jr. 2003).

O tipo e o número de guildas alimentares presentes em uma comunidade varia com as espécies ali existentes, com a densidade de presas e com a resolução taxonômica empregada pelos autores para a classificação das guildas (Angermeier \& Karr 1983, Moyle \& Senanayake 1984). Outros estudos realizados em ambientes tropicais 
e temperados relataram resultados semelhantes aos nossos, sendo que a quantidade de guildas alimentares encontradas varia entre cinco a oito guildas (Peretti \& Andrian 2004, Cassemiro et al. 2005, Rayner 2006).

As planícies inundáveis brasileiras geralmente apresentam um predomínio de espécies detritívoras (Araújo-Lima et al. 1995). Porém, no presente estudo, esse padrão não foi encontrado, apesar de detrito ter sido um item importante na dieta de algumas espécies. Este resultado era esperado já que a maioria das espécies detritívoras são representantes dos Prochilodontidae e Curimatidae (Pereira \& Rezende 1998) que não foram contempladas nas coletas desse estudo.

O predomínio de espécies insetívoras nos ambientes analisados pode ser atribuído ao fato de que diversas espécies de insetos não possuem fortes restrições ambientais. Algumas famílias, como a de Chironomidae, predominam em ambientes aquáticos e mesmo durante secas pronunciadas sua oferta não é limitante (Aburaya \& Calill 2007). Dessa forma, as larvas aquáticas estão entre os recursos mais consumidos por espécies de peixes em ambientes aquáticos tropicais, como relatado por Costa (1987) e Castro \& Casatti (1997).

Neste estudo cinco espécies foram consideradas herbívoras. Esta guilda parece ser exclusiva de ambientes tropicais e subtropicais sul-americanos, não sendo comumente encontrada em comunidades íctícas de regiões temperadas (Winemiller 1991). A herbívora é bastante influenciada pelas variações espaciais e temporais (Goulding 1980) principalmente em áreas alagáveis. As oscilações hidrológicas favorecem um grande crescimento de perifiton e macrófitas aquáticas sobre a vegetação inundada (Resende et al. 1998) permitindo dessa forma o estabelecimento de espécies que exploram esses recursos.

A única espécie pertencente à guilda iliófaga ocorreu no início da enchente, época do ano em que as águas se elevam, as lagoas tendem a estar mais profundas e com maior aporte de sedimentos. No estudo realizado por Gaspar da Luz et al. (2001) a presença de espécies desta guilda também esteve relacionada com a profundidade das lagoas. A presença de espécies zooplanctívoras no início da seca e na enchente pode ser atribuída a dois fatores: i) à retração dos ambientes no período seco, que gerando uma maior densidade de organismos planctônicos, facilita a predação por espécies que se alimentam desses organismos; ii) ao aumento de organismos planctônicos no período de enchente devido a conexão das lagoas isoladas com o corpo d' água principal.

Em nosso estudo apesar das espécies terem explorado os recursos de forma distinta entre os períodos e os locais analisados elas não mudaram de guilda alimentar. Abelha et al. (2001) em uma revisão sobre plasticidade alimentar mostrou que a alimentação da ictiofauna pode sofrer modificações espaciais e temporais, considerando que locais e períodos distintos possuem diferentes condições abióticas, porém essa alteração no espectro alimentar não implica em mudanças na função ecológica das espécies.

Observamos que a riqueza nas guildas alimentares também é afetada pelas características do ambiente sendo que encontramos maior número de espécies dentro de cada guilda no período de águas baixas (junho). Isto pode ser explicado pelo fato das espécies estarem muitas vezes confinadas em habitats totalmente isolados do corpo d'água, o que não possibilita a migração das mesmas para ambientes adjacentes, fazendo com que elas compartilhem os mesmos recursos e guildas alimentares.

Mesmo com uma variação temporal no número de espécies não foi observada uma mudança no número de guildas e de recursos alimentares consumidos. Tal resultado sugere: i) que as espécies inclusas na comunidade ao longo do tempo são agrupadas nas guildas já existentes, sendo que os recursos disponíveis no ambiente são explorados tanto pelas espécies residentes como pelas espécies visitantes; ii) o número de guildas alimentares não varia ao longo do tempo, mas há mudanças na composição das mesmas pois variações temporais no ciclo hidrológico possibilitam a inclusão de novas espécies na comunidade e nas guildas ali presentes. Dessa forma, nossos resultados corroboram com uma regra de assembléia proposta por Fox (1987) e Fox e Brown (1993) que é baseada em grupos funcionais e que sugere que novas espécies que são adicionadas às comunidades tendem a preencher grupos funcionais já existentes.

A presença de algumas guildas somente em determinadas épocas do ano, como por exemplo, da iliófaga apenas no início da enchente, é um resultado comum em estudos de estrutura trófica, sendo que alguns autores atribuem isso a mudanças na disponibilidade de presas no ambiente ao longo do tempo (Little et al. 1998, Hajisamae \& Ibrahim 2008). Winemiller (2006) analisando a variação espacial e temporal em redes tróficas de peixes tropicais da Costa Rica e da Venezuela atribuiu a maior causa da variação temporal nas teias alimentares analisadas à mudanças na disponibilidade e qualidade do habitat $\mathrm{e}$ dos recursos alimentares.

Nossos resultados evidenciaram a persistência de algumas guildas, porém essas apresentaram mudanças em sua composição ao longo do tempo. O aumento, a diminuição ou a substituição de espécies dentro das guildas é um resultado esperado em regiões com alta sazonalidade, como o Pantanal. Isto se deve ao fato de que as interações ecológicas entre as espécies são intensificadas em determinadas épocas, sendo que espécies menos competitivas ou mais susceptíveis à predação são excluídas ou substituídas por espécies com maior adaptabilidade às pressões ambientais.

Outro fator a ser considerado é a estruturação espacial das guildas. Algumas interações interespecíficas podem influenciar tanto os padrões espaciais como os processos dinâmicos da organização da comunidade (Brown et al. 2002). A organização de guildas tróficas dentro de um habitat pode ser vista como uma estratégia para evitar a competição alimentar (Pianka 1980, Angel \& Ojeda 2001) ou para otimizar o uso dos recursos disponíveis no ambiente (Jacksic 1981). As características físicas do habitat são também componentes importantes, já que o tamanho, a distância do corpo d'água, a presença de vegetação entre outras características podem influenciar o tipo e o número de guildas. As espécies podem ocupar locais com maior abundância de recursos mesmo que a captura seja difícil, ou optar por locais com menos recursos de fácil captura (Crowder \& Copper 1982).

A ocorrência de espécies omnívoras, detritívoras, insetívoras, herbívoras e bentívoras em todos os locais analisados sugerem que os representantes dessas guildas possuem ampla distribuição espacial. Diversos trabalhos realizados em diferentes sistemas com estrutura alimentar de peixes relatam a presença dessas guildas (Hahn et al. 1998, Resende 2000, Cunico et al. 2002, Luz-Agostinho et al. 2006). Isto é atribuído às estratégias ecológicas das espécies que permitem que as mesmas possam manter populações viáveis em ambientes com características distintas.

A presença de macrófitas aquáticas nas lagoas analisadas é um fator importante que pode afetar a disponibilidade de recursos para as espécies, observamos que os grupos com maior porcentagem média de macrófitas aquáticas também apresentaram maior número de guildas alimentares. Sabe-se que as comunidades de peixes encontram maior disponibilidade alimentar em locais com macrófitas aquáticas (Rozas \& Odum 1988), pois as macrófitas podem formar substratos onde há o desenvolvimento de organismos que são utilizados na dieta das espécies que ali forrageiam (Junk 1973). Casatti et al. (2003) estudando a importância das macrófitas aquáticas como locais de alimentação para as espécies em um reservatório do rio Paranapanema, também concluíram que os bancos de macrófitas aquáticas servem como um importante micro habitat para o forrageamento de pequenas espécies de peixes. 


\section{Agradecimentos}

Ao CNPq/Programa PELD, ao Centro de Pesquisas do Pantanal (CPP)/Ministério da Ciência e Tecnologia(MTC) e a CAPES pelo apoio financeiro. Á RPPN SESC Pantanal pelo apoio logístico. A Clarissa Moesch Welter e a Rosemary Ferreira Medeiros pelo apoio na identificação dos conteúdos estomacais. Aos revisores pelas críticas e sugestões.

\section{Referências Bibliográficas}

ABELHA, M.C.F, AGOSTINHO, A.A. \& GOULART, E . 2001. Plasticidade trófica em peixes de água doce. Acta Sci. 23(2):425-434.

ABURAYA, F.H. \& CALILL, C.T. Variação temporal de larvas de Chironomidae (Díptera) em um trecho do alto rio Paraguai, Cáceres, MT. 2007. Rev. Bras. Zoo. 24:565-572.

ANGEL, A. \& OJEDA, F.P. 2001.Structure and trophic organization of subtidal fish assemblages on the northern Chilean coast: the effect of habitat complexity. Mar. Ecol. Progr. Ser. 217:81-91. doi:10.3354/meps217081

ANGERMEIER, P.L. \& KARR, J.R. 1983. Fish communities along environmental gradients in a system of tropical streams. Environ. Biol. Fishes. 9:117-135. doi:10.1007/BF00690857

ARAÚJO-LIMA, C.A.R.M., AGOSTINHO, A.A. \& FABRÉ, N.N. 1995. Trophic aspects of fish communities in brazilian rivers and reservoirs. In Limnology in Brazil. (J.B.Tundisi, C.E.M. Bicudo \& T. Matsumura-Tundisi). Brazilian Academy of Science/Brazilian Limnological Society, São Paulo, 105-136.

BAGINSKI, L.J., FLORENTINO, A.C., FERNANDES, I.M., PENHA, J.M.F. \& MATEUS, L.A.F. 2007. A dimensão espacial e temporal da diversidade de peixes da zona litoral vegetada de lagoas marginais da planície de inundação do rio Cuiabá, Pantanal, Brasil. Biota Neotrop. 07(3):233-238. http://www.biotaneotropica.org.br/v9n3/pt/ abstract?article+bn04009032009 (último acesso em: 10/06/2010).

BAILLY, D., AGOSTINHO, A.A. \& SUZUKI, H.I. 2008. Influence of the flood regime on the reproduction of fish species with different reproductive strategies in the Cuiabá River, Upper Pantanal, Brazil. River Res. Appl. 24:1218-1229. doi:10.1002/rra.1147

BICUDO, C.E.M. \& MENEZES, M. 2006. Gênero de Algas de águas Continetais do Brasil.Chave para identificação e descrição. $2^{\text {nd }}$ ed. Rima, São Carlos, 507 p.

BORROR, D.J. \& DELONG, D.M. 1988. Introdução ao estudo dos insetos. Edgard Blucher, Sao Paulo, 653 p.

BROWN, J.H., KELT, D.A. \& FOX, B.J. 2002. Assembly rules and competition in deserts rodents. Amer. Nat. 160(6):815-818. PMid:18707468. doi: $10.1086 / 343882$

BURNS, T.P. 1989. Lindman's contradiction and the trophic structure of ecosystems. Ecology 70:1355-1362. doi:10.2307/1938195

CASATTI, L., MENDES, H.F. \& FERREIRA, K.M. 2003. Aquatic macrophytes as feeding site for small fishes in the Rosana Reservoir. Paranapanema River, Southeastern Brasil. Braz. J. Biol. 63(2):213-222.

CASSEMIRO, F.A., HAHN, N.S. \& FUGI, R. 2005. Estrutura trófica da ictiofauna, ao longo do gradiente longitudinal do reservatório de Salto Caxias (rio Iguaçu, Paraná, Brasil), no terceiro ano após o represamento. Acta Sci. Biol. Sci. 27(1):63-71.

CASTRO, R.M.C. \& CASATTI, L. 1997. The fish fauna from a small forest stream of the upper Paraná River basin, southeastern Brazil. Ichthyol. Explor. Fresh. 7(4):337-352.

CATELLA, A.C. 1992. Estrutura da comunidade e alimentação de peixes da Baía da Onça, uma lagoa do Pantanal do rio Aquidauana, MS. Dissertação de mestrado, Universidade Estadual de Campinas, Campinas.

CLARKE, K.R. 1993. Non-parametric multivariate analysis of changes in community structure. Aus. J. Ecol. 18:117-143. doi:10.1111/j.1442-9993.1993.tb00438.x

COSTA, W.J.E.M. 1987. Feeding habits of a fish community in a tropical Coastal stream, Rio Mato Grosso, Brazil. Stud. Neotropica Fauna \& Environment. 22:145-153. doi:10.1080/01650528709360728
CROWDER, L.B. \& COOPER, W.E. 1982. Habitat structural complexity and the interaction between bluegills and their prey. Ecology. 63:1802-1813. doi: 10.2307/1940122

CUNICO, A.M., GRAÇA, W.J., VERISSIMO, S. \& MAURICIO, L. 2002. Influencia do nível hidrológico sobre a assembléia de peixes em lagoa sazonalmente isolada da planície de inundação do alto rio Paraná. Acta Scienctiarum, 24(2):383-389.

DEUS, C.P. \& PETRERE JR. 2003. Seasonal diet shifts of seven fish species in atlantic rainforest stream in Southeastern Brazil. Braz. Jour. Biol. 63(4):579-588.

DUFFY, J.E., RICHARDSON, J.P. \& FRANCE, K. E. 2005. Ecosystem consequences of diversity depend on food chain length in estuarine vegetation. Ecol. Lett. 8:301-309. doi:10.1111/j.1461-0248.2005.00725.x

ELLIOTT, M., WHITFIELD, A.K., POTTER, I., BLABER, S.J.M., CYRUS, D.P., NORDLIE, F.G. \& HARISSON, T.D. 2007. The guild approach the categorizing estuarine fish assemblages: a global review. Fish and Fisheries. 8:241-268. doi:10.1111/j.1467-2679.2007.00253.x

ELMOOR-LOUREIRO, L.M.A. 1997. Manual de identificação de cladóceros límnicos do Brasil. Universa, Brasília.

FOX, B.J. 1987. Species assembly and the evolution of community structure. Evol. Ecol. 1: 201-213. doi:10.1007/BF02067551

FOX, B.J. \& BROWN, J.H. 1993. Assembly rules for functional groups in North American desert rodent communities. Oikos. 67:358-370. doi: $10.2307 / 3545483$

GASPAR DA LUZ, K.D., ABUJANRA, F., AGOSTINHO, A.A. \& GOMES, L.C. 2001. Caracterização trófica da ictiofauna de três lagoas da planície aluvial do alto rio Paraná, Brasil. Acta Sci. 23(2):401-407.

GOULDING, M. 1980. The fishes and the forest: explorations in amazon natural history. University of California Press, Berkeley, 280 p.

GRENOUILLET, G. \& PONT, D. 2001. Juvenile fishes in macrophyte beds: influence of food resources, habitat structure and body size. Jour. Fish. Biol. 59:939-959. doi:10.1111/j.1095-8649.2001.tb00163.x

HAHN, N.S., AGOSTINHO, A.A., GOMES, L.C. \& BINI, L.M. 1998. Estrutura trófica da ictiofauna do reservatório de Itaipú (Paraná, Brasil) nos primeiros anos de sua formação. Interc. 23(5):299-305.

HAJISAMAE, S. 2009. Trophic ecology of bottom fishes assemblage along coastal areas of Thailand. Estuarine, Coastal and Shelf Science. 82:503-514. doi:10.1016/j.ecss.2009.02.010

HAJISAMAE, S., CHOU, L.M. \& IBRAHIM, S. 2003. Feeding habitats and trophic organization of the fish community in shallow waters of $n$ impacted tropical habitat. Est. Cos. and She. Sci. 58:89-98. doi:10.1016/ S0272-7714(03)00062-3

HAJISAMAE, S. \& IBRAHIM, S. 2008. Seasonal and spatial variations of fish trophic guilds in a shallow, semi-enclosed tropical estaurine bay. Environ. Biol. Fish. 82:251-264. doi:10.1007/s10641-007-9278-6

HOEINGHAUS, D.J., WINEMILLER, K.O. \& AGOSTINHO, A.A . 2008. Hydromorphology and river impoundment affect food-chain length of diverse neotropical food webs. Oikos, 117:984-995. doi:10.1111/j.00301299.2008.16459.x

HOMMER, O., HARPER, D.A.T. \& RYAN, P.D. 2001. Past: Paleontological statistics software package for education and data analysis. Paleontol. Elet. 4(1):9.

HYNES, H.B.N. 1950. The food of freshwater sticklebacks (Gasterosteus aculeatus and Pigosteus pungitius), with a review of methods used in studies of the food of fishes. J. Anim. Ecol. 19(1):411-429.

JACKSIC, F.M. 1981. Abuse and misuse of the term "guild" in ecological studies. Oikos. 83: 87-92.

JUNK, W.J. 1973. Investigations on the ecology and production biology of the "floating meadows" (Paspalo-Echinochloetum) on the middle Amazon. II. The aquatic fauna in the root-zone of floating vegetation. Amazon. 4:9-102.

KOPP, M.M. , DE SOUZA, V.Q., COIMBRA, J.L.M, LUZ, V.K., MARINI, N. \& OLIVEIRA, A.C. 2007. Melhoria da correlação cofenética pela exclusão de unidades experimentais na construção de dendogramas. Rev. Fac. Zoo. Vet. e Agr. 14(2):46-53. 
LAKE, P.S. 2003. Ecological effects of pertubation by drougth in flowing waters. Freshw. Biol. 48:1161-1172. doi:10.1046/j.13652427.2003.01086.x

LITTLE, S.A., TONN, W.M., TALLMAN, R.F \& REIST, J.D. 1998. Seasonal variation and trophic relationships within the fish communities of the lower Slave River, Northwest Territories, Canada. Environ. Biol. Fish. 53:429-445. doi:10.1023/A:1007433400648

LUZ-AGOSTINHO, K.D.G., BINI, L.M., FUGI, R., AGOSTINHO, A.A \& JR, H.F.J. 2006. Food spectrum and trophic structure of the ichthyofauna of Corumbá reservoir, Paraná river Basin, Brazil. Neotrop. Ichthyol. 4:61-68.

MENGE, B.A., LUBCHENCO, J., ASHKENAS, L.R. \& RAMSEY, F. 1986. Experimental separation os effects of consumers on sessile prey in the low zone of rocky shore in the bay of Parana: direct and indirect consequences of food web complexity. J. Exp. Mar. Biol. Ecol. 100:225-269. doi:10.1016/0022-0981(86)90164-4

MEYER, A.S. 2002. Comparação de coeficientes de similaridade usados em análises de agrupamento com dados de marcadores moleculares dominantes. Dissertação de mestrado, Universidade de São Paulo.

MOYLE, P.B. \& SENANAYAKE, F.R. 1984. Resource partitioning among the fishes of rainforest streams in Sri Lanka. Jour. Zool. 202:195-223. doi:10.1111/j.1469-7998.1984.tb05951.x

NEEDHAM, J.G. \& NEEDHAM, P.R. 1982. Guía para el estudio de los seres vivos de lãs aguas dulces. Barcelona, Ed. Reverte, 82 p.

PACE, M.L., COLE, J.J., CARPENTER, S.R., KITCHELL, J.F., HODGSON, J.R., BOGERT, M.C.V., BADE, D.L., KRITZBERG, E.S. \& BASTVIKEN, D. 2004. Whole-lake carbon-13 additions reveal terrestrial support of aquatic food webs. Nature. 427:240-243. PMid:14724637. doi:10.1038/nature02227

PADOVANI, C.R., PADOVANI, S.L.A.G. \& BRANDÃO, M.F. 2003. Mosaico de imagens de satélite do Pantanal no ano de 2000. Corumbá, $18 \mathrm{p}$.

PAINE, R.T. 1980. Food webs: linkage, interaction strength and community infrastructure. J. Anim. Ecol. 49: 667-685. doi:10.2307/4220.

PEREIRA, R.A.C. \& RESENDE, E.K. 1998. Peixes detritívoros da planície inundável do rio Miranda, Pantanal, Mato Grosso do Sul, Brasil. EMBRAPA/CPAP. 12:50

PERETTI, D. \& ANDRIAN, I.F. 2004. Trophic structure of fish assemblages in five permanent lagoons of the high Parana River floodplain, Brazil. Environ. Biol. Fishes. 71:95-103. doi:10.1023/ B:EBFI.0000043155.76741.a1

PEREZ, G.R. 1988. Guia para el estudio de los macroinvertebrados acuaticos del Departamento de Antioquia. Conciencias, Fen Colombia, 217 p.

PIANKA, E.R. 1980. Guild structure in desert lizards. Oikos. 35:194-201. doi: $10.2307 / 3544427$
RAYNER, T.S. 2006. The trophic ecology of the freshwater fishes of an Australian rainforest river. Tese de doutorado, School of Marine and Tropical Biology - James Cook University, Australia.

RESENDE, E.K. 1996. Trophic Structure of Fish Assemblages in the Lower Miranda River, Pantanal, Mato Grosso Do Sul State, Brazil. Rev. Bras. Biol. 60(3):389-403.

RESENDE, E.K. 2000. Trophic structure of fish assemblages in the Lower Miranda river, Pantanal, Mato Grosso do Sul State, Brazil. Rev. Bras. Biol. 60(3):389-403.

RESENDE, E.K., PEREIRA, R.A.C \& ALMEIDA, V.L.L. 1998. Peixes herbívoros da planície inundável do rio Miranda, Pantanal, Mato Grosso do Sul, Brasil. EMBRAPA/CPAP. 10: 24.

ROMESBURG, H.C. 1985. Cluster analysis for researchers. Lifetime Learning Publications, Belmont.

ROSS, S.T. 1986.Resource partitioning in fish assemblages: a review of field studies. Copeia. 2:352-358. doi:10.2307/1444996

ROZAS, L.P. \& ODUM, W.E. 1988. Occupation of submerged aquatic vegetation by fishes:testing the roles of food and refuge. Oecologia. 77:101-106. doi:10.1007/BF00380932

SAZIMA, I. \& MACHADO, F.A. 1990. Underwater observations of piranhas in western Brazil. Environ. Biol. Fishes. 28:17-31. doi:10.1007/ BF00751026

SIMBERLOFF, D. \& DAYAN, T. 1991.The guild concept and structure of ecological communities. Ann. Rev. Ecol. Syst. 22:115-143. doi:10.1146/ annurev.es.22.110191.000555

SOKAL, R.R. \& ROHLF, F.G. 1995. Biometry. $3^{\text {nd }}$ ed. Freeman andCompany, New York.

SPECZIÁR, A. \& REZSU, E.T. 2009. Feeding guilds and food resource partitioning in a lake fish assemblage: an ontogenetic approach. J. Fish Biol. 75:247-267. PMid:20738494. doi:10.1111/j.10958649.2009.02283.x

STEHR, F.W. 1987. Immature Insects. Publishing Hunt Publishing Company, Dubuque, $754 \mathrm{p}$.

WILKINSON, L. 2000. Systat: the system for statistics. Evanston, Illinois.

WINEMILLER, K.O. 1991. Ecomorphological diversification in lowland freshwater fish assemblages from five biotic regions. Ecol. Monogr. 61(4):343-365. doi:10.2307/2937046

WINWMILLER, K.O. 2006. Spatial and temporal variation in tropical fish trophic networks. Ecol. Monogr. 60(3):331-367.

WINEMILLER, K.O. \& JEPSEN, D.B. 1998. Effects of seasonality and fish movement on tropical river food webs. J. Fish Biol. 53:267-296. doi:10.1111/j.1095-8649.1998.tb01032.x

YODZIS, P. 1982. The compartmentation of real na assembled ecossystms. Am. Nat. 120:551-570. doi:10.1086/284013.

Recebido em 04/07/2010

Versão reformulada recebida em 23/02/2011

Publicado em 07/03/2011 